\title{
Incorporating directionality in the modelling of firebreaks using a two-dimensional dynamic fire spread simulator
}

\author{
$\underline{\text { William Swedosh }}^{\mathrm{a}}$ and James Hilton ${ }^{\mathrm{b}}$ \\ ${ }^{a}$ Land \& Water, CSIRO, Clayton, VIC 3168, Australia \\ ${ }^{b}$ Data61, CSIRO, Clayton, VIC 3168, Australia \\ Email:Will.Swedosh@csiro.au
}

\begin{abstract}
Accurate predictions of wildfires are necessary for planning and operational fire management applications. While operational predictive models are aimed at capturing the effect of weather, vegetation and topography on fire spread, the understanding and incorporation of disruptions in the landscape such as firebreaks, roads and rivers are less understood. Developing and implementing models that capture the effect of disruptions on the spread of fires in the landscape is likely to lead to more accurate predictions of fire spread.

Studies have shown that the probability of fires crossing firebreaks depends on factors such as the fire behaviour, the width of the disruption and type and condition of the vegetation the fire is burning through. While computational fluid dynamics models show some promise at simulating breaching firebreaks, they are quite complicated and too slow to be included in operational predictions. Empirical models are far simpler and are much more suitable for fast simulations.

In this study we implement an empirical firebreak breaching model using the Spark wildfire simulator. We extend the model to include local fireline intensity and the wind direction relative to disruption using an effective width implementation. We also develop and utilise a method to include wind bearing fluctuations in the simulations.

We tested our implementation against the empirical model for fires approaching firebreaks of varying widths. There was complete agreement between the empirical model and whether or not the firebreak was breached in the simulation. We then tested the effective width implementation with simulations of fires approaching a firebreak at different angles. Again, there was complete agreement between the result from the empirical model and the fire simulation on what approach angle was required to stop the fire breaching the firebreak due to an increasing effective firebreak width.
\end{abstract}

We then conducted simulations for more realistic scenarios using a road network as the firebreak where the road network had sections at different angles to the firebreak. The simulation results showed differences in behaviour with road orientation, with head on approaches able to breach the road, while some sufficiently angled roads were unable to be breached. Wind fluctuations were included in the final simulation, which allowed the fire to cross the road in more locations than before.

We intend to build upon the methods developed here so that more realistic firebreak breaching can be modelled in operational simulations in the future.

Keywords: Wildfire, modelling, firebreak, disruption 


\section{INTRODUCTION}

Un-burnable areas disrupt the spread of a wildfire by slowing or even stopping the propagation of flames. Such disruptions can be either artificially created, such as a firebreak or area of applied fire retardant, or a part of the existing landscape, such as a river, road, or areas of sand or bare rock. Disruptions are a critical factor for the overall growth, spread and eventual size of a wildfire and must be correctly taken into account in wildfire spread models. Whereas large-scale disruptions are straightforward to implement (such as areas of no fuel or bodies of water), narrow disruptions (such as roads and firebreaks) present some modelling challenges as it may not be obvious whether they are likely to stop the fire. Furthermore, few experiments studies have been carried out to investigate fire propagation over narrow disruptions.

Wilson (1988) showed that the probability of fires crossing firebreaks depends on factors such as the fire behaviour, the width of the disruption and type and condition of the vegetation the fire is burning through. This was determined from a set of experiments in which grass plots of $100 \mathrm{~m}$ by $100 \mathrm{~m}$ were set alight and allowed to burn up to firebreaks of various widths. The findings from these experiments were developed into empirical relationships, which can be incorporated into operational wildfire models due to their relative simplicity. More recent computational fluid dynamics (CFD) studies have shown that whether a fire crosses a firebreak depends on the width of the disruption, the burning fuel characteristics, the local slope of the terrain and the local meteorological conditions (Bellemare et al., 2007 and Morvan, 2015). However, the fine grain inputs and the computational time required to run such models mean they are unlikely to be used in operational wildfire models in the near future.

Developing and implementing models that capture the critical influence of disruptions on wildfires could provide improved predictions of fire spread and the opportunity to test and develop suppression strategies. Swedosh et al. (2017) explored several deterministic and probabilistic implementations of empirical models in wildfire simulations where it was found that deterministic methods could be used to approximate probabilistic ensemble results. The purpose of this paper is to further this previous study by investigating the relationship between the orientation of the firebreak to the spread of the fire. This is likely to be an important factor when calculating the probability of firebreak breach. It should be noted that in this study we are considering the disruption crossing process seen in grasslands and croplands where flame contact is the main driver of the process (Wilson 1988), and not heavy spotting as can occur in forest like vegetation with significant elevated and bark fuel loads as this can be considered as a separate type of dynamic behaviour.

\section{METHODOLOGY}

\subsection{Empirical model used}

In this study we decided to implement an empirical disruption crossing model due to the ease of computation in a wildfire simulator. We chose the model from Wilson (1988) as an example where the probability of breaching the firebreak is given by a logistic function $f$ :

$$
p_{\text {breach }}=f\left(I, w, P_{T}\right)
$$

where $I$ is fireline intensity (Byram 1959), $w$ is firebreak width (perpendicular crossing distance) and $P_{T}$ is the presence of trees near the firebreak. For this study, we assumed at least one tree is present near all firebreaks.

The model was implemented in such a way that if the probability of breaching the firebreak was greater than 0.5 , then the firebreak would be breached. This gives a 'best estimate' of where a fire is likely to spread in a simulation, however if a user of the model / software (such as a land manager) wanted to be more conservative, then they could choose a lower threshold probability of crossing (or even zero to ignore them).

\subsection{Directionality of firebreak}

To model cases where the fire approaches a firebreak at an oblique angle (rather than perpendicularly), certain assumptions had to be made. The first assumption was that the wind is the main driver of disruption breaching, and that disruptions are always crossed in the direction of the wind (this assumption may be reviewed in future work when slopes of different aspects are included). It then follows that an oblique fire approach angle results in a larger effective disruption width for the fire to overcome. This effective width is visualised in Figure 1 and quantified in Equation (2):

$$
w_{e f f}=\frac{w}{\boldsymbol{n}_{F b} \cdot \widehat{\boldsymbol{u}}}
$$


where $w$ is the perpendicular firebreak width, $\boldsymbol{n}_{F b}$ is the unit normal vector of the firebreak pointing away from the approaching fire and $\widehat{\boldsymbol{u}}$ is the unit vector in the direction of the wind. The effective width should then be used instead of the perpendicular width to calculate breach probability in (1). Adjustments to this methodology, including setting a maximum effective width multiplier, will be addressed in future work.

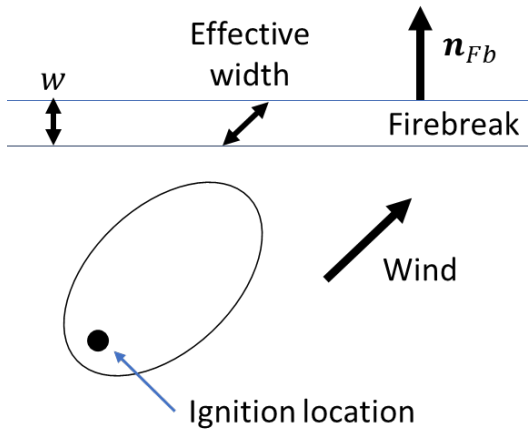

Figure 1. A larger effective firebreak width is caused by the driving wind not being lined up with the normal to the firebreak. Elliptical 'wind driven' fire perimeter shown in black.

Another factor to consider when the fire approaches a firebreak at an oblique angle, is that the local fireline intensity around the fire perimeter will be smaller than the fireline intensity at the head of the fire (Catchpole et al. 1982). In this study, we chose to calculate the local fireline intensity using the rate of spread in the direction normal to the local fire perimeter as per Catchpole et al. 1982. Other methods, such as using rate of spread in the direction of the ignition location to the perimeter, may be considered in future work.

Accounting for fluctuations in wind direction may also be desirable operationally as wind is known to fluctuate about its mean bearing. This was incorporated as an optional parameter in the simulation set up, where the fluctuation angle was used to recalculate the minimum $w_{\text {eff }}$ and maximum local fireline intensity within the fluctuation envelope to conservatively calculate the highest breach probability. For example, if the wind direction and firebreak normal are misaligned by $20^{\circ}$, implementing a $5^{\circ}$ wind bearing fluctuation means that we consider a minimum misalignment of $15^{\circ}$ which gives a lower effective width. Similarly, we would recalculate the local rate of spread (as per Catchpole et al. 1982) using a misalignment between the wind direction and the normal of the fire perimeter of $5^{\circ}$ less (capped at $0^{\circ}$ ), which gives a greater fireline intensity.

\subsection{Simulation implementation}

In this study, the Spark wildfire modelling framework (Miller et al. 2015) was used to implement the firebreak crossing methodologies. Spark is a two-dimensional, raster-based level set solver with the capability to allow individual raster cells to be un-burnable or to burn with a user-defined rate of spread. The state can be switched from 'burnable' to 'un-burnable' or vice versa based on user-defined criteria.

A schematic diagram of the implementation for disruptions is shown in Figure 2. At the initialisation state, all raster cells within the domain are set to the 'burnable' state. Disruptions can be manually defined or imported into the model as either vector or raster layers. Next, a check is carried out for any cells intersecting with a disruption, which are then set to the 'un-burnable' state. For the validation part of the study, the disruption is manually defined as a line of grids cells (with a constant width), but for the road crossing test case, the disruption is read in as a vector of the road network (with the road width defined as an attribute of the network).

During the simulations, the fire perimeter is propagated and any cells adjacent to the fire are checked to see if they are in the disruption layer in an 'un-burnable' state. In this implementation of the disruption model, the state of the cell is switched to 'burnable' if the probability of breach exceeds 0.5 in that cell.

The simulations themselves were run at a grid size of 10 metres. For disruptions with widths greater or smaller than 10 metres, the exact width of the disruption was assigned to the disruption cell as an attribute. This allows us to resolve and model disruptions of any reasonable width. Grid size sensitivity checking will be done in future work.

The fire was propagated using the McArthur Mk V head fire rate of spread (McArthur 1973), translated to equations by Noble et al. (1980), with an elliptical template used to calculate rate of spread around the perimeter from the head fire rate of spread (Catchpole et al. 1982). There was no build-up phase implemented in this study. This will be included in future work where it can be used to reduce the rate of spread for a fixed time after ignition or reduce the rate of spread of a fire after it breaches a disruption. 


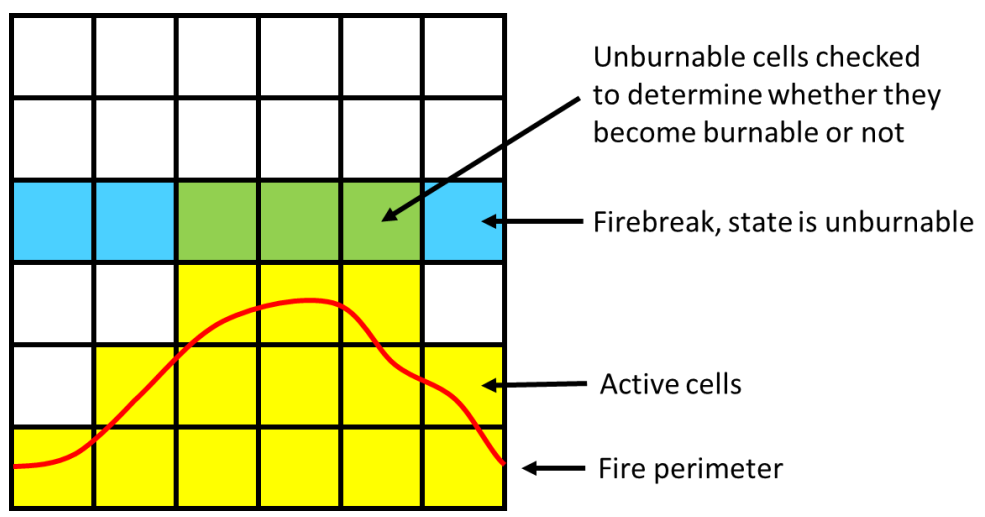

Figure 2. A diagram showing a fire approaching a row of firebreak cells. Yellow cells are burning or have previously burned, blue cells are currently un-burnable, green cells are being checked to see whether they become burnable or not.

The manually defined validation cases (firebreak width and angle variations) were run for a 1.5-hour simulation from a circular ignition, while the road network test case was run for a 1-hour simulation from a line ignition. The validation cases had fuel loads and weather conditions of $25 \mathrm{t} \mathrm{ha}^{-1}$ and a Forest Fire Danger Index (FFDI) of 80 such that the peak fireline intensity for the head fire was $31 \mathrm{MW} \mathrm{m}^{-1}$.

For the road network simulations, the distance from the road at each simulation cell and the bearing of each road segment were precalculated for simulation efficiency, while the road width was read directly from the road network shapefile. These simulations had fuel loads and weather conditions of $7.55 \mathrm{t} \mathrm{ha}^{-1}$ and an FFDI of 100 such that the peak fireline intensity for the head fire was $3.5 \mathrm{MW} \mathrm{m}^{-1}$.

\section{RESULTS}

\subsection{Disruption width variation}

To test the implementation of the model of Wilson (1988) in the Spark simulation software, a set of simulations was conducted where the disruption width was varied. We used a threshold crossing probability of 0.5 to implement the model deterministically (i.e., a crossing probability of greater than 0.5 would allow the fire to breach the disruption). The results of six different disruption widths are shown in Figure 3 where it can be seen the simulation with no disruption showed no resistance in propagating, and as the disruption width increased the proportion of the fire able to cross the disruption decreased due to less of the fire perimeter having the required intensity to do so.

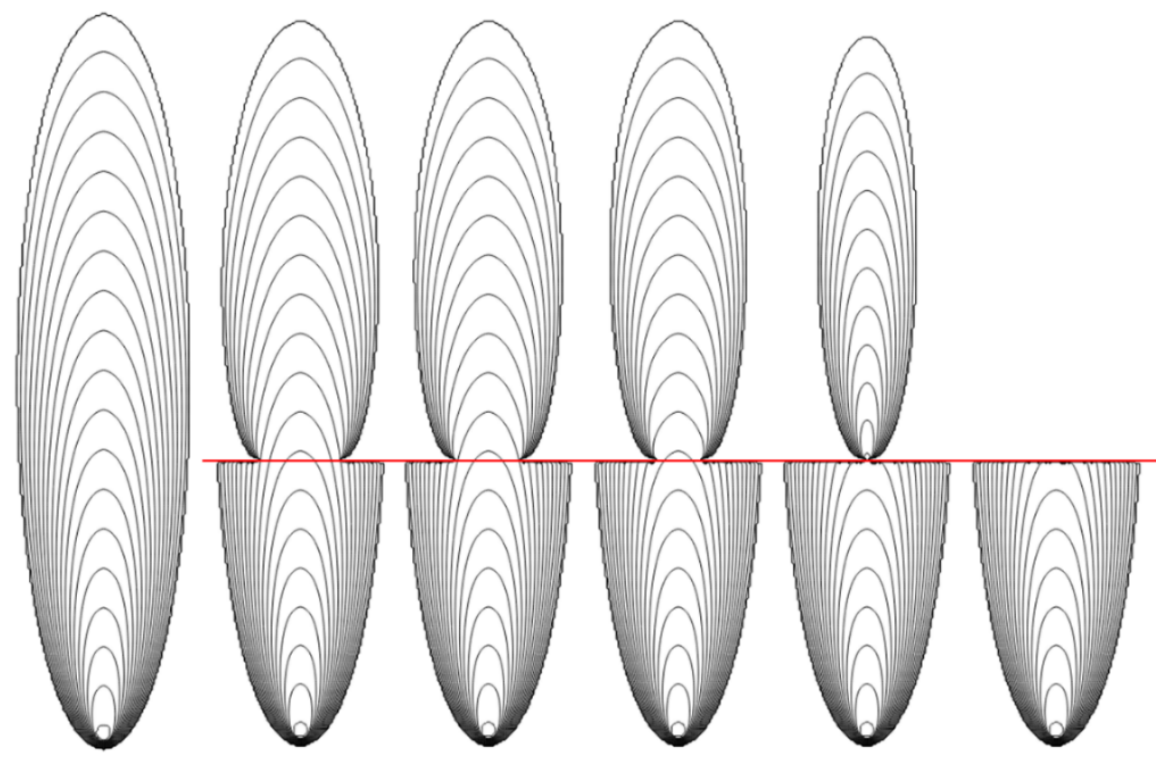

Figure 3. Simulations of wildfires approaching disruptions of various widths. From left to right, disruptions have widths of $0,14.8,20.9,27.2,32.9$ and 33 metres with respective head fire crossing probabilities of 1 , $0.999,0.99,0.9,0.51$ and 0.49 .5 -minute isochrones shown in black, disruption location shown in red. 
To verify that the basic model was working as expected, two specific disruption widths were chosen as $32.9 \mathrm{~m}$ and $33.0 \mathrm{~m}$, as these widths corresponded to head fire crossing probabilities of 0.51 and 0.49 respectively. From Figure 3 when the disruption width was $32.9 \mathrm{~m}$, only the very head of the fire had the required intensity to breach, whereas when the disruption was $33.0 \mathrm{~m}$ it did not. This result strongly indicates that the breach probabilities have been calculated and implemented correctly.

\subsection{Disruption approach angle variation}

To verify that the effective disruption width was being implemented correctly, a set of simulations were conducted where the fire approach angle was varied from 0 to 70 degrees. For these simulations, a disruption width of $14.8 \mathrm{~m}$ (corresponding to a head fire crossing probability of 0.999 in the previous section) was kept constant, resulting in effective widths ranging from 14.8 to 43.3 metres. The results of these simulations are shown in Figure 4 where the fire is able to breach the firebreak for the simulations where the effective firebreak width is less than the critical 33 metres identified in the previous section required to stop a similar fire.

For further validation, two more simulations were run with approach angles of 63 and 64 degrees, corresponding to effective disruption widths of 32.6 and 33.8 metres (Figure 5). Encouragingly, the simulations behaved as expected, with crossing probabilities of 0.53 and 0.42 respectively allowing the fire to breach and be held up by the firebreak.

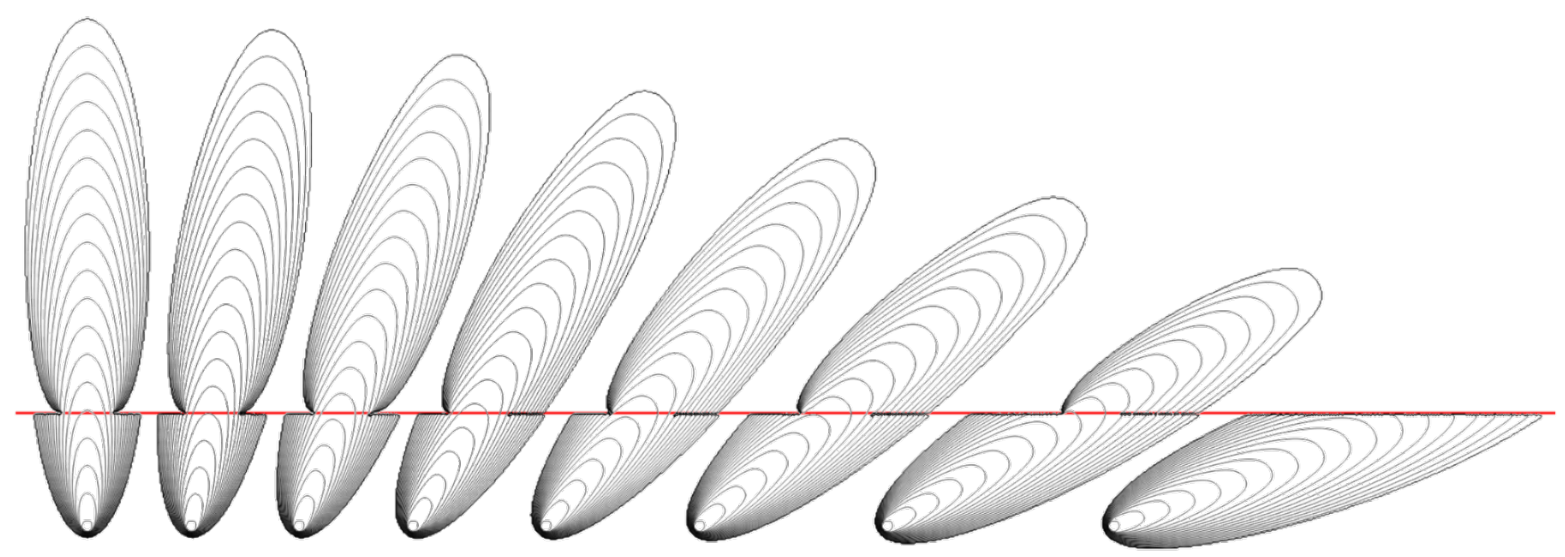

Figure 4. Simulations of wildfires approaching a $14.8 \mathrm{~m}$ wide disruption at varying angles. From left to right, angle offsets are $0,10,20,30,40,50,60$ and 70 degrees with respective effective firebreak widths of 14.8, 15.0, 15.7, 17.1, 19.3, 23.0, 29.6 and 43.3 metres. 5-minute isochrones shown in black, disruption location shown in red.

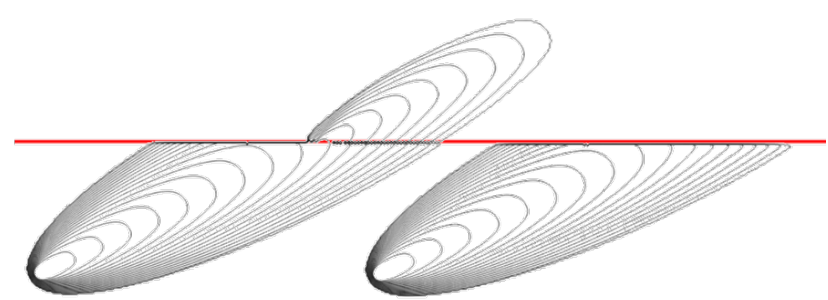

Figure 5. Simulations of wildfires approaching a $14.8 \mathrm{~m}$ wide disruption at $63^{\circ}$ and $64^{\circ}$ angles (left to right). The simulations have respective effective firebreak widths of 32.6 and 33.8 metres. 5 -minute isochrones shown in black, disruption location shown in red.

\subsection{Road network example}

Given the successful testing of the implementation of the model, a more realistic scenario was simulated with a continuous road that changed its bearing along its length. In this scenario, a line ignition was used so that the head fire would be broader than from a point ignition and have a consistent fireline intensity, allowing the influence of road bearing to be seen more clearly. All sections of road have an equal width of 6.8 metres in this location.

Figure 6 shows the results of the simulation. The road in region B is nearly perpendicular to the wind direction, meaning that the effective width of the road is minimised, and the fire can cross as it has sufficient intensity 
( $p_{\text {breach }}$ of 0.51). Similarly, the road in region $\mathrm{D}$ and $\mathrm{F}$ is not angled enough to increase the effective width above the critical width ( $7 \mathrm{~m}$ in this case) of the fire at its current intensity ( $p_{\text {breach }}$ of 0.50 to 0.51 ). The road in regions $\mathrm{C}$ and $\mathrm{E}$ is angled enough to increase the effective width above the critical width, stopping the fire ( $p_{\text {breach }}$ of 0.47 to 0.49 ). The roads in sections $\mathrm{A}$ and $\mathrm{G}$ stop the fire as it is not the true head of the fire that is impacting the road, rather the fire moving around to the flank where the fire burns with a lower intensity which is not able to overcome the road width ( $p_{\text {breach }}$ of 0.30 to 0.49 ).

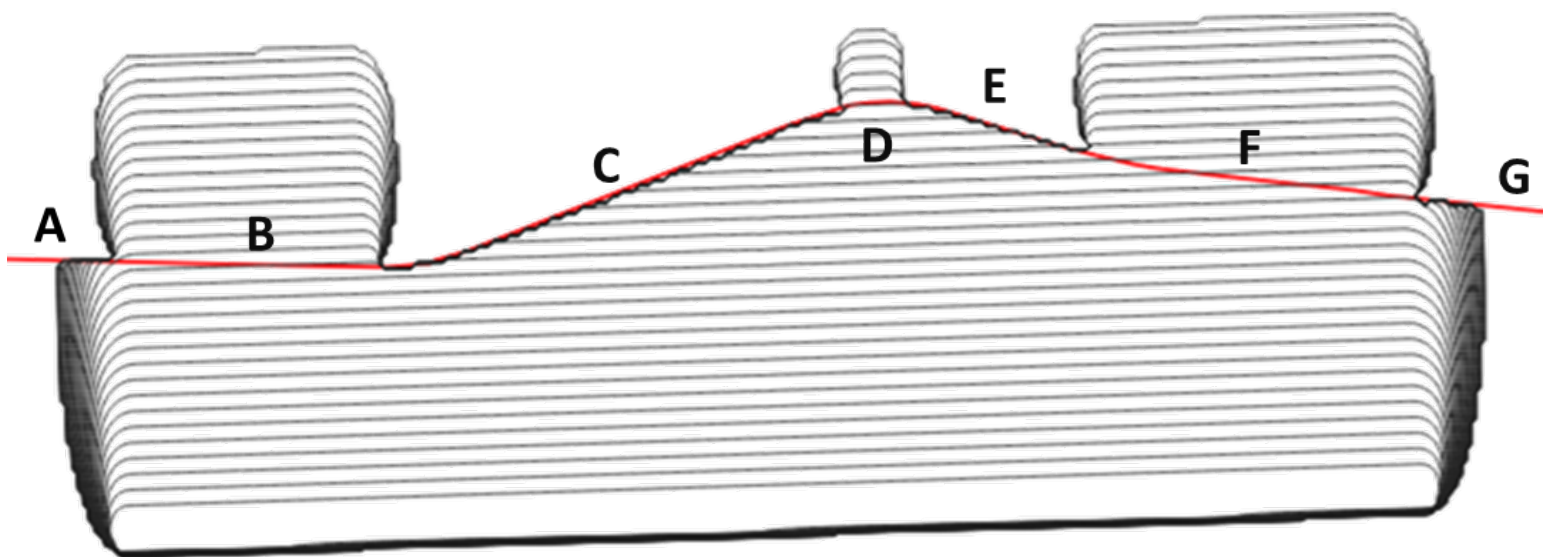

Figure 6. A simulation of a line ignition fire approaching a 6.8 metre wide road at varying orientations. 2minute isochrones shown in black, disruption location shown in red.

Another simulation was run, this time including a $5^{\circ}$ wind bearing fluctuation as described in Section 2.2. As expected, including this assumption allowed the effective width to be reduced, and the local fireline intensity to increase enough for the fire to breach the road in more locations. Figure 7 shows that the fire was now able to breach region E where it wasn't able to without wind bearing fluctuations, and that region $\mathrm{D}$ is breached over a larger length. The head fire is now only predicted to be stopped by the road in region $\mathrm{C}$ where the approach angle is the largest. The roads in sections $\mathrm{A}$ and $\mathrm{G}$ are sill able to stop the fire due to being impacted by the flanking fire with lower local fireline intensity. Interestingly, the road between $\mathrm{C}$ and $\mathrm{D}$ allows the fire to breach a road which has a very similar bearing to the road in region $\mathrm{C}$ which stops the fire. This is most likely due to the bearing of the road being precalculated at a grid resolution of 10 metres, which has the potential to include small numerical errors. This phenomenon will be investigated in future work which will include a grid resolution study.

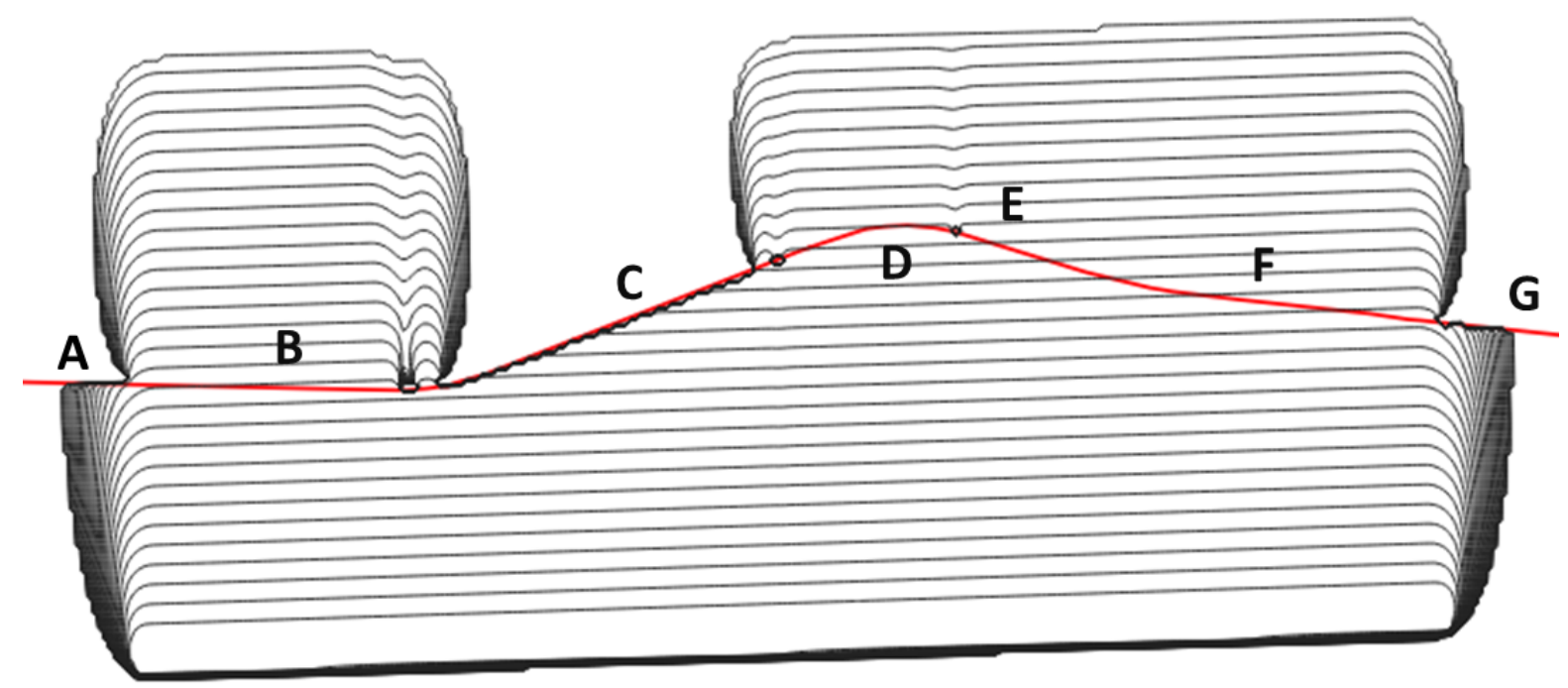

Figure 7. A simulation of a line ignition fire approaching a 6.8 metre wide road at varying orientations. $5^{\circ}$ wind fluctuation incorporated. 2-minute isochrones shown in black, disruption location shown in red. 


\section{DISCUSSION AND CONCLUSION}

We have developed a deterministic method of implementing empirical firebreak crossing probability functions in a two-dimensional fire spread simulator. The method was verified against the source equations for a simple scenario where an elliptical fire approached a firebreak head on. We then extended this method to include oblique approach angles by considering the effective firebreak width, and again evaluated our results with the empirical model.

Our model implementation was then trialled for a more realistic road crossing scenario where it was shown that multiple road segments of different bearings could be modelled in a single simulation. Our model was extended again to include a potential wind fluctuation which could account for some of the uncertainty associated with forecast wind directions. Including wind fluctuations allowed the fire to cross the road in more locations and is one way that a user of this model could add conservatism to the fire simulations.

Ideally, the implementations explored in this study could make their way into operational tools where emergency managers could use them for either live fire prediction or planning applications. The specific application could inform the parameters used in the model. For example, when assessing a potential prescribed burn, control lines of varying widths could be assessed to reduce the probability of breach to below an acceptable threshold. Another example could include running a best estimate operational prediction for where the fire is likely to go (by selecting a threshold breach probability of 0.5 ) and comparing that to a prediction where the threshold breach probability is set to 0.01 or 0 to quantify how significant it may be if specific firebreaks are breached.

Future work will include exploring adjustments and enhancements to this methodology including setting a maximum effective width multiplier and investigating firebreak breaching in the direction of fire spread (rather than just in the wind direction), which is likely important when slope effects are involved. A grid resolution study will also be conducted to determine whether there is any sensitivity of numerical error to grid cell size.

Expanding this methodology to include capturing the likelihood of firebreak breaching due to short range spotting is also of interest.

\section{REFERENCES}

Bellemare L.O., Porterie B., Loraud J.C. (2001), On the Prediction of Firebreak Efficiency, Combustion Science and Technology, 163, 131-176

Byram, G.M. (1959) Combustion of forest fuels. In 'Forest fire: control and use.' (Ed. KP Davis.) pp. 61-89. (McGraw-Hill: New York)

Catchpole, E.A., de Mestre, N.J., Gill, A.M. (1982) Intensity of fire at its perimeter. Australian Forest Research 12, 47-54

McArthur A.G. (1973), Forest Fire Danger Meter Mark V. Commonwealth Department of National Development Forestry and Timber Bureau, Canberra, ACT.

Miller C., Hilton J., Sullivan A., Prakash M. (2015), SPARK - A Bushfire Spread Prediction Tool. ISESS 2015. IFIP Advances, vol 448. Springer, Cham

Morvan D. (2015), Numerical study of the behaviour of a surface fire propagating through a firebreak built in a Mediterranean shrub layer, Fire Safety Journal, 71, 34-48

Noble I.R., Bary G.A.V. and Gill A.M. (1980), McArthur's fire-danger meters expressed as equations. Australian Journal of Ecology 5, 201-203.

Swedosh W., Hilton J., Prakash M. (2017), Modelling Firebreaks in a Two Dimensional Dynamic Fire Spread Simulator, MODSIM2017, 22nd International Congress on Modelling and Simulation, pp. 1180-1186

Wilson A.A.G. (1988), Width of firebreak that is necessary to stop grass fires: some field experiments. Canadian Journal of Forest Research, 18, 682-687 